\title{
Enfrentamiento del tabaquismo en el paciente hospitalizado
}

\author{
LISANDRO STUARDO T.* y CLAUDIA BAMBS S.**
}

\section{Smoking cessation in the hospitalized patient}

Hospitalization is a valuable opportunity for smoking cessation. In Chile this opportunity is wasted. The need to hospitalize is determined by diseases often caused by smoking, this scenario allows the patient to assess not only the consequences of this addiction, but also the importance of stopping tobacco consumption. During hospitalization, the nicotine withdrawal syndrome appears, whose recognition and management is essential to avoid habitual complications: anxiety or delirium. For all of the above mentioned reasons, it is necessary the adequate confrontation of smoking in the hospitalized patient, which is recognized by international accreditation bodies as Joint Commission. A Cochrane metaanalysis 2012 concluded that the two pillars of effective interventions were the multimodal character (counseling and pharmacological treatment), and ambulatory follow-up for more than one month after discharge. The central elements of a brief counseling can be summarized in ABC, where A: ask about smoking; B: give brief advice stating the importance of quitting, and C: provide support for cessation for motivated patients. Although the above-mentioned interventions involve resources for pharmacological support, there seems to be no excuse for implementing simple interventions in Chilean hospitals, such as documenting the smoking status of each patient and offering brief counseling.

Key words: Smoking; smoking cessation; hospitalization; counseling; Substance Withdrawal Syndrome.

\section{Resumen}

La hospitalización es una oportunidad valiosa para el abandono del tabaquismo, desaprovechada en Chile. La necesidad de hospitalizarse está determinada por enfermedades muchas veces causadas por el consumo de tabaco, este escenario permite al paciente valorar no solo las consecuencias de esta adicción, si no también, la importancia de suspender el consumo. Es esperable que durante la hospitalización aparezca el síndrome de abstinencia de nicotina, cuyo reconocimiento y manejo es fundamental para evitar complicaciones habituales: ansiedad o delirium. Por todo lo anterior, resulta necesario el adecuado enfrentamiento del tabaquismo en el paciente hospitalizado, lo que es reconocido por organismos acreditadores internacionales como Joint Commission. Un metanálisis Cochrane 2012 concluyó que los dos pilares fundamentales de las intervenciones efectivas fueron el carácter multimodal (consejería y tratamiento farmacológico), y el seguimiento ambulatorio por más de un mes posterior al alta. Los elementos centrales de una consejería breve pueden resumirse en el $A B C$, siendo A: averiguar sobre el consumo de tabaco; $B$ : dar un consejo breve indicando la importancia de dejar de fumar, y C: Ofrecer apoyo para la cesación a los pacientes que se muestren motivados. Si bien las intervenciones mencionadas involucran contar con recursos para apoyo farmacológico, no pareciera haber excusas para seguir sin implementar en los hospitales chilenos intervenciones sencillas como documentar el estado tabáquico de cada paciente y ofrecer consejería breve.

Palabras clave: Fumar; dejar de fumar; hospitalización; sindrome de abstinencia.

* Departamento de Enfermedades Respiratorias del Adulto, Facultad de Medicina, Pontificia Universidad Católica de Chile.

** Departamento de Salud Pública y Centro Avanzado de Enfermedades Crónicas ACCDiS (Fondap 15130011). Escuela de Medicina, Pontificia Universidad Católica de Chile. 
La hospitalización ofrece una oportunidad valiosa para el cese del tabaquismo, pero que es habitualmente desaprovechada en Chile. La necesidad de hospitalizarse está determinada por enfermedades con grados variables de riesgo vital y muchas veces con directa relación al consumo de tabaco, como patología cardiovascular, respiratoria u oncológica, por mencionar algunas. Este escenario determina una situación particularmente sensible en donde el paciente puede valorar de forma concreta no solo las consecuencias del hábito tabáquico, si no también, la importancia de suspender el consumo. Para muchos, la hospitalización constituirá motivación suficiente para realizar un intento de cesación ${ }^{1}$. Por otro lado, según la Ley N. ${ }^{\circ} 20.660$, artículo 11 , los hospitales chilenos son recintos $100 \%$ libres de humo, donde el paciente se encuentra libre de los gatillantes sociales que favorecen el consumo. Además, existe acceso directo a apoyo por parte del equipo de salud para educación y tratamiento. Asimismo, es esperable que durante la estadía hospitalaria se manifieste el síndrome de abstinencia de nicotina, cuyo reconocimiento y manejo es fundamental para evitar complicaciones habituales como ansiedad o delirium. Por todo lo anterior, resulta necesario el adecuado enfrentamiento del tabaquismo en el paciente hospitalizado. Así lo han reconocido organismos acreditadores internacionales como Joint Commission, e instituciones canadienses y españolas que han desarrollado modelos de intervención en cesación tabáquica iniciadas en el hospital.

\section{Intervenciones multimodales iniciadas en el paciente hospitalizado}

Se han conducido diversos estudios con pacientes fumadores hospitalizados. Un metanálisis Cochrane 2012 concluyó que los dos pilares fundamentales de las intervenciones efectivas fueron el carácter multimodal (incluyendo la consejería y tratamiento farmacológico) y el seguimiento ambulatorio por más de un mes posterior al alta ${ }^{2}$.

En relación con la consejería, ésta debe ser realizada durante la hospitalización, por cualquier miembro del equipo de salud capacitado para este fin (médicos, enfermeras, TENS, kinesiólogos, nutricionistas u otros). Según el modelo propuesto en la guía neozelandesa de cesación tabáquica, los elementos centrales de una consejería breve pueden resumirse en el $\mathrm{ABC}$, siendo A: averiguar sobre el consumo de tabaco; B: dar un consejo breve indicando la importancia de dejar de fumar, y C: Ofrecer apoyo para la cesa- ción a los pacientes que se muestren motivados. Algunas particularidades del $\mathrm{ABC}$ en pacientes hospitalizados incluyen la relevancia de dejar un registro sobre el consumo de tabaco en la ficha del paciente, y el vincular el consejo breve al motivo de hospitalización ${ }^{3}$.

Por su parte, el tratamiento farmacológico considera no solo la terapia de reemplazo (parches y chicles de nicotina) para el período de abstinencia $^{4}$, sino también el tratamiento ambulatorio de mantención (bupropión, vareniclina $\mathrm{u}$ otro) al alta $^{5}$.

El seguimiento posterior debe incluir un canal de contacto que se mantenga por lo menos durante un mes, ya sea en formato presencial o vía correo electrónico, mensajes de texto, Quit-lines $o$ sistemas automatizados de voz (Interactive voice response $=I V R$ ).

En la evaluación de la eficacia de las intervenciones de cesación tabáquica en pacientes hospitalizados destacan los estudios HAND-1 y HAND-2, ambos estudios randomizados y controlados, liderados por Rigotti y cols. El estudio HAND-16 incluyó a 397 pacientes fumadores hospitalizados. El grupo intervención involucró tratamiento farmacológico financiado por el estudio y seguimiento por 3 meses. La terapia farmacológica se escogía en conjunto con el paciente y podía corresponder a reemplazo de nicotina (parches o chicles), bupropión, vareniclina, o una combinación de ellos. El seguimiento se realizó por vía telefónica a través de un sistema IVR en el que se evaluaba la disponibilidad, adherencia y tolerancia al tratamiento farmacológico. Se realizaba, además, refuerzo positivo y se ofrecía la posibilidad de contactar directamente a un profesional. El grupo control, en cambio, recibía recomendación de tratamiento farmacológico que debía ser financiado por el mismo paciente, y la sugerencia de contactar una Quit-Line estatal gratuita. Ambos grupos recibieron consejería breve durante la hospitalización. Los resultados demostraron una diferencia significativa en abstinencia de tabaco a los 6 meses de $27 \%$ en el grupo intervención y $16 \%$ grupo control (RR 1,7; IC $95 \% 1,1-2.5, \mathrm{p}=0,007$ ).

Posteriormente, se realizó el estudio HAND- $2^{7}$ que mantuvo el diseño del HAND-1, pero en un contexto multicéntrico reuniendo un total de 1300 participantes. Sorprendentemente, no se encontraron diferencias significativas entre ambos grupos en la abstinencia de tabaco a 6 meses, con $17 \%$ en el grupo intervención y $16 \%$ en el grupo control $(\mathrm{p}=0,58)$. Al comparar los estudios HAND 1 y 2 se concluyó que no existieron diferencias en las intervenciones de consejería y tratamiento far- 
macológico, pero sí en el sistema de seguimiento, que en el caso del estudio HAND-2 presentó fallas del sistema IVR para facilitar la entrega de terapia farmacológica y no permitía contacto con el equipo investigador. El análisis de ambos estudios enseña que no cualquier intervención de cesación tabáquica intrahospitalaria resultará efectiva, y que el carácter multimodal y un adecuado diseño del seguimiento son elementos clave para el éxito de una intervención de este tipo.

\section{Iniciativas internacionales: \\ Joint Commission, Modelo de Ottawa y Modelo Español}

La importancia de la hospitalización como ventana de oportunidad para la cesación tabáquica ha motivado cambios en los estándares de cuidados de los prestadores y acreditadores de salud a nivel mundial. La Joint Commission es una organización americana que acredita a prestadores de salud en los Estados Unidos y en el resto del mundo. Desde 2012 esta comisión exige un conjunto de medidas de desempeño para múltiples patologías, entre ellas el manejo del paciente tabáquico hospitalizado donde se detalla: a) documentar el estado de fumador/no fumador de todos los pacientes que ingresan a la institución; b) entregar consejería y farmacoterapia; c) derivar al momento del alta a un profesional que entregue consejería y prescriba terapia farmacológica de mantención durante el período de cesación; y d) documentar la condición de uso de tabaco 30 días posterior al alta hospitalaria ${ }^{1}$.

El modelo canadiense, por su parte, busca abordar el tratamiento de la dependencia al tabaco tanto en un contexto ambulatorio como hospitalario y es conocido como el modelo de la Universidad de Ottawa (Ottawa Model for Smoking Cessation $=\mathrm{OMSC}^{8}$. Esta iniciativa data desde 2002 y su propósito ha sido aumentar la proporción de pacientes que logran suspender la adición al tabaco de forma permanente posterior a un evento de salud que requiere hospitalización. Para conseguirlo se estableció la necesidad de identificar de forma sistemática a todos los pacientes fumadores al momento de su ingreso hospitalario, posteriormente ofrecer consejería dirigida a los beneficios del abandono del tabaco por parte de un profesional entrenado, y proveer tratamiento farmacológico para manejar la abstinencia durante la hospitalización y para tratamiento a largo plazo. Al momento del alta los pacientes son ingresados a un sistema automatizado de seguimiento tipo IVR, que se encarga de contactar al paciente por vía telefónica y con una interfaz de voz digitalizada pregrabada, en 8 oportunidades a lo largo de los 6 meses post alta (a los 3, 14, 30, $60,90,120,150$ y 180 días). En cada ocasión se le pregunta al paciente si se ha mantenido abstinente, si ha usado los medicamentos, y se le entrega refuerzo positivo; en caso de que el paciente mencione haber recaído o que se siente en riesgo de recaer, el sistema ofrece contacto directo con una enfermera parte del equipo. Cada llamada tiene una duración de $3 \mathrm{~min}$, y las respuestas de los pacientes son digitalizadas e ingresadas a una base de datos. El análisis de la efectividad del modelo fue publicado el año 2010, en donde al comparar 2 grupos de pacientes (275 en grupo intervención y 360 controles) se encontró una mejoría en la abstinencia continua a los 6 meses en el grupo intervención ( $29.4 \%$ versus $18.3 \%$, OR $1,71,95 \%$ IC $1.1-2.6, p=0,02)^{9}$. Lo que originalmente se planteó como una intervención local en la Provincia de Ontario, se extendió luego al resto del país. Una publicación reciente muestra el impacto del modelo de Ottawa tras 2 años de implementación del sistema, consiguiendo una disminución significativa en el número de hospitalizaciones y en la mortalidad a un año post alta ${ }^{10}$.

A nivel de habla hispana, destaca la nueva Normativa para el Tratamiento del Tabaquismo en Pacientes Hospitalizados de la Sociedad Española de Neumología y Cirugía Torácica, que coincide en la relevancia de implementar los elementos clave identificados en los modelos americano y canadiense, con énfasis en contar con un protocolo de seguimiento más allá de un mes post alta ${ }^{11}$.

\section{Mensaje final}

El enfrentamiento del tabaquismo en pacientes hospitalizados es un problema complejo, sin embargo, se ha conseguido dar un primer paso al establecer los elementos clave de una intervención exitosa.

Si bien muchas de las intervenciones mencionadas involucran contar con recursos para apoyo farmacológico, no pareciera haber excusas para seguir sin implementar en los hospitales chilenos intervenciones sencillas como documentar el estado tabáquico de cada paciente y ofrecer consejería breve. Cabe destacar la importancia de la detección de comorbilidades psiquiátricas, particularmente trastornos de ansiedad o de ánimo, cuyo tratamiento adecuado puede mejorar de forma significativa tanto la calidad de vida como 
las posibilidades de dejar de fumar.

Para el seguimiento post alta resulta muy oportuno el uso de tecnologías móviles como Smartphones y Tablets para promover el automanejo y contacto con el equipo de salud en caso necesario. Asimismo, las tecnologías móviles pueden permitir el envío de refuerzos positivos y la pesquisa precoz de aquellos pacientes en riesgo de recaer, los cuales pueden beneficiarse de una evaluación personalizada ${ }^{12}$.

En lo inmediato, el desafío es contar con evidencia local sobre intervenciones de cesación tabáquica iniciadas en hospitales chilenos, así como generar conciencia entre nuestros pares y tomadores de decisión sobre la relevancia de convertir la "crisis de la hospitalización" en una "oportunidad única para la cesación tabáquica".

\section{Bibliografía}

1.- FIORE MC, GOPLERUD E, SCHROEDER SA. The Joint Commission's New Tobacco-Cessation Measures - Will Hospitals Do the Right Thing?. N Engl J Med 2012; 366: 1172-4.

2.- RIGOTTI NA, CLAIR C, MUNAFÒ MR, et al. Interventions for smoking cessation in hospitalised patients. Cochrane Database Syst Rev 2012; 5: CD001837.

3.- MCROBBIE H, BULLEN C, GLOVER M, WHITTAKER R, WALLACE-BELL M, FRASER T. New Zealand Smoking Cessation Guidelines. N Z Med J 2008; 121(1276): 57-70.

4.- RIGOTTI NA, ARNSTEN JH, MCKOOL KM, WOOD-REID KM, SINGER DE, PASTERNAK RC. The use of nicotine-replacement therapy by hospitalized smokers. Am J Prev Med 1999; 17: 255-9.

5.- SMITH BJ, CARSON KV, BRINN MP, LABISZE-
WSKI NA, PETERS MJ, FITRIDGE R, et al. Smoking Termination Opportunity for inPatients (STOP): superiority of a course of varenicline tartrate plus counselling over counselling alone for smoking cessation: a 12-month randomised controlled trial for inpatients. Thorax 2013; 68: 485-6.

6.- RIGOTTI NA, REGAN S, LEVY DE, JAPUNTICH S, CHANG Y, PARK ER, et al. Sustained care intervention and postdischarge smoking cessation among hospitalized adults: a randomized clinical trial. JAMA 2014; 20; 312: 719-28.

7.- RIGOTTI NA, TINDLE HA, REGAN S, LEVY DE, CHANG Y, CARPENTER KM, et al. A Post-Discharge Smoking-Cessation Intervention for Hospital Patients: Helping Hand 2 Randomized Clinical Trial. Am J Prev Med 2016; 51: 597-608.

8.- REID RD, PIPE AL, QUINLAN B. Promoting smoking cessation during hospitalization for coronary artery disease. Can J Cardiol 2006; 22: 775-80.

9.- REID RD, MULLEN KA, SLOVINEC D'ANGELO ME, AITKEN DA, PAPADAKIS S, HALEY PM, et al. Smoking cessation for hospitalized smokers: an evaluation of the "Ottawa Model". Nicotine Tob Res. 2010; 12(1): 11-8.

10.- MULLEN KA, MANUEL DG, HAWKEN SJ, E PIPE AL, COYLE D, HOBLER LA, et al. Effectiveness of a hospital - initiated smoking cessation programme: 2 year health and healthcare outcomes. Tobacco Control 2017 26:293-9. doi: 10.1136/tobaccocontrol-2015-052728

11.- JIMÉNEZ C, GRANDA J, SOLANO S. Normativa sobre el tratamiento del tabaquismo en pacientes hospitalizados - Sociedad Española de Neumología y Cirugía de Tórax (en prensa).

12.- WHITTAKER R, MCROBBIE H, BULLEN C, et al. Mobile phone-based interventions for smoking cessation. Cochrane Database Syst Rev 2012; 11: CD006611.

Correspondencia a:

Dra. Claudia Bambs S.

Email: cbambs@uc.cl 